\title{
Influência da réplica experimental nos parâmetros estimados para os modelos cinéticos da produção de etanol em batelada
}

\author{
Zanardi, M. S. ${ }^{1 *}$; Costa Jr., E.F. ${ }^{1}$ \\ 1 Programa de Pós-Graduação em Engenharia Química, Universidade Federal do Espírito Santo, Alegre, ES, Brasil \\ *e-mail: mayarazanardi@outlook.com
}

\begin{abstract}
Resumo
Modelos matemáticos são importantes para analisar o comportamento do processo para diferentes condições operacionais. A modelagem do processo fermentativo é complexa e os modelos cinéticos podem conter diversos parâmetros. Os parâmetros podem ser obtidos na literatura ou estimados por meio da resolução de um problema de otimização, cuja função objetivo é, por exemplo, o somatório dos quadrados dos resíduos (diferença entre o valor simulado e experimental). Todo experimento está sujeito a erros de medida e estes erros podem influenciar, em maior ou menor grau, os valores dos parâmetros estimados. Este trabalho tem como objetivo determinar o efeito dos erros experimentais nos parâmetros dos modelos cinéticos para 0 processo de produção de etanol. Os parâmetros foram estimados empregando-se a implementação do método Simplex Nelder-Mead no Matlab. Os dados utilizados na investigação foram resultantes de ensaios experimentais em batelada com diferentes amostras de substratos, obtidos da literatura. As amostras de substratos foram obtidas em duplicata de diferentes empresas. Como resultado, além da esperada diferença nos parâmetros cinéticos, obtidos para as amostras de diferentes empresas, também foram observadas diferenças para os parâmetros da mesma empresa, ao se empregar réplicas.
\end{abstract}

\begin{abstract}
Mathematical models are important to analyze the process behavior for different operational conditions. The modeling of the fermentation process is complex and kinetic models can contain different parameters. The parameters can be obtained from the literature or can be estimated by solving an optimization problem whose objective function is for example the sum of squares of the residuals (difference between the simulated and experimental value). All experiments are subject to measurement error and these errors may affect, to a greater or lesser extent, the values of estimated parameters. The proposal of this paper is to determine the effect of experimental errors on the parameters of kinetic models for the ethanol production process. The parameters were estimated using the Matlab implementation of the Nelder-Mead Simplex. The data used in this work are from the literature and are related to batch experiments with different substrates. Samples of substrates were obtained in duplicate from different companies. As a result, in addition to the expected difference in the kinetic parameters obtained for samples of different companies, differences were also observed between the parameters estimated for each replica of the samples of same company.
\end{abstract}

Keywords (Palavras chaves): modelagem, otimização, estimação de parâmetros.

\section{Introdução}

A produção de etanol por via fermentativa é a mais econômica e difundida no Brasil. Qualquer produto que contenha carboidrato constitui-se de matéria-prima para a obtenção de etanol. No Brasil as matériasprimas principais utilizadas são a cana-de-açúcar e o mel (subproduto da produção de açúcar) [1-2]. 
O processo de fermentação pode ser representado por duas equações principais em condições anaeróbias. A primeira etapa consiste na hidrólise da sacarose pela enzima invertase, Equação 1, e a segunda etapa é a reação de fermentação simplificada, Equação 2.

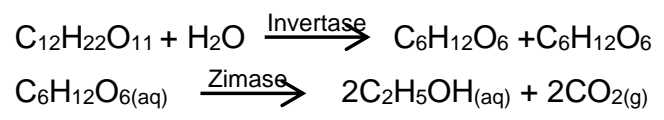

A modelagem matemática é importante para prever o comportamento de um processo e as respostas do sistema à mudanças nas variáveis do processo, permitindo aperfeiçoá-lo. A modelagem do processo fermentativo é complexa, devido às inúmeras reações químicas e bioquímicas que ocorrem, envolvendo várias fases e um sistema com múltiplos componentes. O cálculo da velocidade específica de crescimento dos microrganismos $(\mu)$ é o primeiro passo para uma boa formulação e ajuste do modelo matemático, é na construção da equação cinética que reside toda a dificuldade da formulação dos modelos fenomenológicos dos processos fermentativos [3-4].

A equação mais simples para se determinar à velocidade específica de crescimento dos microrganismos é a de Monod (Equação 3). Esse modelo explica a relação entre a concentração do substrato limitante no meio, com a velocidade específica de reprodução do microrganismo.

$$
\mu=\mu_{\max } \cdot \frac{\mathrm{S}}{\mathrm{K}_{\mathrm{s}}+\mathrm{S}}
$$

Onde $\mu_{\max }$ é a taxa máxima de crescimento celular, $\mathrm{S}$ a concentração do substrato e $K_{s}$ representa a concentração de substrato em que a velocidade de crescimento é metade da velocidade máxima.

A equação de Monod descreve adequadamente a cinética de fermentação, porém não é viável quando há componentes metabólicos tóxicos que inibem 0 crescimento celular. A ausência da inibição é uma situação incomum na prática, principalmente durante um cultivo descontínuo, onde há um crescente acúmulo de metabólitos que acabam interferindo desfavoravelmente sobre o metabolismo e crescimento microbianos [4-5].

Outros diversos modelos com diferentes graus de complexidade foram desenvolvidos ao longo do tempo.

A Tabela 1 apresenta alguns modelos cinéticos que levam em consideração algum tipo de inibição [6].

Os modelos cinéticos podem conter diversos parâmetros, dependendo da complexidade do modelo. A estimativa desses parâmetros é uma das etapas do processo de formulação do modelo matemático do processo fermentativo. Os valores desses parâmetros podem ser obtidos na literatura ou ser estimados através de um algoritmo de otimização.

Todo experimento está sujeito a erros de medida e estes erros podem influenciar, em maior ou menor grau, os valores dos parâmetros estimados [7]. Este trabalho tem como objetivo determinar o efeito dos erros experimentais nos parâmetros dos modelos cinéticos para o processo de produção de etanol.

Tabela 1: Modelos cinéticos.

\begin{tabular}{|c|c|c|c|c|}
\hline & Modelo & Característica & & Equação \\
\hline$\mu=\mu_{\max }$ & $\frac{\mathrm{S}}{\mathrm{K}_{\mathrm{S}}+\mathrm{S}+\frac{\mathrm{S}^{2}}{\mathrm{~K}_{\mathrm{i}}}} \cdot\left(1-\frac{\mathrm{P}}{\mathrm{P}_{\max }}\right.$ & $\begin{array}{c}\text { Substrato limitante e } \\
\text { inibição pela concentração } \\
\text { de substrato e produto }\end{array}$ & GHOSE e TYAGI (1979) & (4) \\
\hline$\mu=\mu_{\max }$ & $\frac{\mathrm{S}}{\mathrm{K}_{\mathrm{s}}+\mathrm{S}} \cdot\left(1-{\frac{\mathrm{P}}{\mathrm{P}_{\max }}}^{\mathrm{n}}\right.$ & $\begin{array}{c}\text { Substrato limitante e } \\
\text { inibição pela concentração } \\
\text { de produto }\end{array}$ & LEVENSPIEL (1980) & (5) \\
\hline$\mu=\mu_{\max }$ & $\cdot \mathrm{e}^{-\mathrm{K}_{1} \cdot \mathrm{P}-\mathrm{K}_{2} \cdot \mathrm{S}} \cdot \frac{\mathrm{S}}{\mathrm{K}_{\mathrm{s}}+\mathrm{S}}$ & $\begin{array}{c}\text { Substrato limitante e } \\
\text { inibição pela concentração } \\
\text { de substrato e produto }\end{array}$ & JIN et al. (1981) & (6) \\
\hline$\mu=\mu_{\max } \cdot \frac{S}{K_{s}+S}$ & $\cdot\left(1-{\frac{\mathrm{P}}{\mathrm{P}_{\max }}}^{\mathrm{n}} \cdot\left(1-{\frac{\mathrm{X}}{\mathrm{X}_{\max }}}^{\mathrm{m}}\right.\right.$ & $\begin{array}{c}\text { Substrato limitante e } \\
\text { inibição pela concentração } \\
\text { de células e produto }\end{array}$ & LEE et al. (1983) & (7) \\
\hline$\mu=\mu_{\max } \cdot \frac{S}{K_{s}+S}$ & 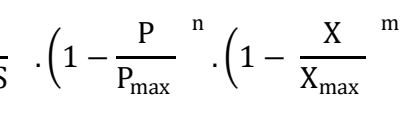 & $\begin{array}{c}\text { Substrato limitante e } \\
\text { inibição pela concentração } \\
\text { de substrato e produto }\end{array}$ & TOSETTO (2002) & (8) \\
\hline
\end{tabular}




\section{Materiais e métodos}

O conjunto de parâmetros dos modelos apresentados na Tabela 1 é estimado para quatro diferentes ensaios experimentais em batelada, obtidos na literatura. As amostras de substrato foram obtidas em duplicata de duas empresas diferentes. A nomenclatura para as duas empresas, adotada no presente trabalho, é o uso das siglas ALV (de Minas Gerais) e BEN (de São Paulo), seguidas do número do experimento (igual a 1 ou 2, já que tem-se réplicas para cada empresa) [6].

Os parâmetros são estimados separadamente, para cada uma das réplicas experimentais, por meio da resolução de um problema de otimização. A função objetivo a ser minimizada é o somatório dos quadrados dos resíduos (diferença entre o valor simulado e experimental) para a concentração de células (X), concentração de substrato (S) e concentração produto $(\mathrm{P})$, conforme definido na Equação 9.

$$
\begin{aligned}
\operatorname{minimizar} f(\mathbf{p})= & { }_{\mathrm{i}=1}^{\mathrm{n}}\left(\mathrm{X}_{\mathrm{e}, \mathrm{i}}-\mathrm{X}_{\mathrm{s}}(\mathbf{p})_{\mathrm{i}}\right)^{2}+\left(\mathrm{S}_{\mathrm{e}_{\mathrm{i}}}-\mathrm{S}_{\mathrm{s}}(\mathbf{p})_{\mathrm{i}}{ }^{2}\right. \\
& +\left(\mathrm{P}_{\mathrm{e}_{\mathrm{i}}}-\mathrm{P}_{\mathrm{S}}(\mathbf{p})_{\mathrm{i}}{ }^{2}\right.
\end{aligned}
$$

Onde $X_{\text {ei }}, S_{\text {ei }}$ e $P_{\text {ei }}$ são os valores experimentais de $X, S$ e $P$ no tempo ti, $\mathbf{p}$ é o vetor que contém as variáveis do problema de otimização, ou seja, cada um dos parâmetros do modelo cinético considerado e $X_{s}(\mathbf{p})$, $S_{s}(\mathbf{p})$ i e $P_{s}(\mathbf{p})$ i são os valores simulados de $X$, S e $P$ no tempo ti, calculados a partir da integração numérica do sistema de EDOs formado pelas Equações 10 à 12 do tempo inicial $(t=0)$ até o tempo $t_{i}$. Os modelos, Equações 10 à 12, foram desenvolvidos em função dos balanços de conservação de massa e os fatores de conversão (Equação 13).

$$
\begin{aligned}
& \frac{d X}{d t}=r_{x}=X \cdot \mu_{X} \\
& \frac{d S}{d t}=-r_{s}=-\frac{1}{Y_{X / S}} \cdot X \cdot \mu_{X} \\
& \frac{d P}{d t}=r_{p}=\frac{Y_{P} / S}{Y_{X / S}} \cdot X \cdot \mu_{X} \\
& r_{x}=X \cdot \mu_{X}=r_{S} \cdot Y_{X / S}=r_{P} \cdot \frac{Y_{P / S}}{Y_{X / S}}
\end{aligned}
$$

Onde $\mathrm{Y}_{\mathrm{x} / \mathrm{s}}$ é o rendimento de células e $\mathrm{Y}_{\mathrm{p} / \mathrm{s}} \mathrm{O}$ rendimento em etanol, representados respectivamente nas Equações 14 e 15. O valor desses parâmetros varia de acordo com as condições industriais, os valores utilizados no trabalho foram obtidos na literatura [6].

$$
\begin{gathered}
Y_{P / S}=\frac{P-P_{0}}{S_{0}-S} \\
Y_{X / S}=\frac{X-X_{0}}{S_{0}-S}
\end{gathered}
$$

O sistema de EDOs a ser resolvido não possui solução analítica e deve ser resolvido por meio de integração numérica. Esta integração numérica é efetuada pelo método de Runge-Kutta de $4^{\mathrm{a}}$ ordem. O problema de otimização é resolvido utilizando o método Simplex Nelder-Mead, ambos no software Matlab. Com os parâmetros estimados, para cada réplica experimental, uma discussão sobre o efeito a influência da réplica

\begin{tabular}{|c|c|c|c|c|c|c|c|c|c|c|c|c|}
\hline & \multicolumn{4}{|c|}{ JIN } & \multicolumn{4}{|c|}{ GHOSE } & \multicolumn{3}{|c|}{ LEVENSPIEL } & \multirow[b]{2}{*}{$f(p)$} \\
\hline & Ks & $\mathrm{K}_{1}$ & $\mathrm{~K}_{2}$ & $f(\mathbf{p})$ & $\mathrm{K}_{\mathrm{s}}$ & $\mathrm{K}_{\mathrm{i}}$ & $P_{\max }$ & $f(\mathbf{p})$ & $\mathrm{K}_{\mathrm{s}}$ & $\mathrm{K}_{\mathrm{i}}$ & $\mathrm{n}$ & \\
\hline ALV.1 & 61,06 & 0,0142 & 0,0139 & 91,29 & 232,17 & 14,90 & 650,11 & 101,71 & 2190,39 & 186,15 & $-3,9$ & 92,69 \\
\hline ALV.2 & 163,35 & 0,0059 & 0,0112 & 332,33 & 323,51 & 16,87 & 58790,25 & 331,68 & 2153,80 & 381,93 & $-8,14$ & 332,12 \\
\hline BEN.1 & 387,22 & 0 & 0,0108 & 536,76 & 462,90 & 11,10 & $1,28 \times 10^{14}$ & 559,34 & 3030,65 & $1,97 \times 10^{6}$ & $-5,55 \times 10^{4}$ & 533,96 \\
\hline BEN.2 & 420,42 & 0 & 0,0105 & 479,50 & 505,50 & 11,01 & $7,01 \times 10^{14}$ & 502,67 & 3009,25 & $1,10 \times 10^{6}$ & $-3 \times 10^{4}$ & 477,58 \\
\hline
\end{tabular}
experimental é realizada.

\section{Resultados e discussão}

Nas Tabelas 2 e 3 estão apresentados os resultados da estimação dos parâmetros cinéticos dos quatro ensaios utilizando os cinco diferentes modelos apresentados na Tabela 1.

Tabela 2: Resultado da estimação dos parâmetros para os modelos de Jin, Ghose e Levenspiel. 
Tabela 3: Resultado da estimação dos parâmetros para os modelos de Tosetto e Lee.

\begin{tabular}{|c|c|c|c|c|c|c|c|c|c|c|c|}
\hline & \multicolumn{5}{|c|}{ TOSETTO } & \multicolumn{6}{|c|}{ LEE } \\
\hline & $\mathrm{K}_{\mathrm{s}}$ & $\mathrm{K}_{\mathrm{i}}$ & $P_{\max }$ & $\mathrm{n}$ & $f(p)$ & $\mathrm{K}_{\mathrm{s}}$ & $P_{\max }$ & $\mathrm{n}$ & $X_{\max }$ & $\mathrm{m}$ & $f(p)$ \\
\hline ALV.1 & 230,38 & 14,89 & 378,28 & 0,5828 & 101,70 & 2190,16 & 186,15 & $-3,91$ & $2,65 \times 10^{5}$ & 1,57 & 92,69 \\
\hline ALV.2 & 323,71 & 16,87 & 390,74 & 0 & 331,68 & 2153,68 & 381,94 & $-8,14$ & $2,25 \times 10^{4}$ & 0,72 & 332,11 \\
\hline BEN.1 & 462,92 & 11,06 & 1159,16 & 0 & 559,34 & 3030,33 & $1,01 \times 10^{5}$ & $-2828,96$ & $7,88 \times 10^{8}$ & $-51,32$ & 533,99 \\
\hline BEN.2 & 505,52 & 11,01 & 93,57 & 0 & 502,67 & 3009,38 & $1,39 \times 10^{6}$ & $-3,79 \times 10^{4}$ & $9,44 \times 10^{10}$ & $-7,38$ & 477,57 \\
\hline
\end{tabular}

Pode-se observar nas Tabelas 2 e 3 que há uma diferença significativa, para todos os parâmetros de todos os modelos, entre os valores obtidos para a empresa ALV e BEN. Considerando-se o parâmetro $\mathrm{K}_{\mathrm{s}}$, presente em todos os modelos, a maior diferença (cerca de $260 \%$ ) entre o valor médio para as duas réplicas de uma empresa e de outra, foi para o modelo de Jin, cujo Ks médio para ALV foi de 112,2 e para BEN de 403,8. Ainda para este parâmetro, a menor diferença entre as médias foi obtida para o modelo de Levenspiel (2172 contra 3020). Mesmo neste caso, a diferença (cerca de 30\%) é significativa. Esta diferença é esperada já que diferentes empresas utilizam diferentes méis. A causa desta alteração nos méis se deve às especificidades das condições operacionais de produção de açúcar e à diferença na cana-de-açúcar empregada (espécie e/ou local de produção).

Também pode ser constatada nas Tabelas 2 e 3 uma variação nos valores estimados para uma mesma empresa considerando-se apenas o efeito da réplica experimental. Ainda se analisando o parâmetro Ks, a diferença devido à réplica é maior (quase 168\%) para o modelo de Jin, adotando-se os dados da empresa ALV. Entretanto, esta variação superior a $100 \%$ só é obtida neste caso, sendo que em algumas situações ela praticamente não ocorre (modelos de Lee e Levenspiel).

O conjunto de parâmetros obtidos de cada amostra é muito especifico. Por exemplo, se forem empregados os parâmetros da amostra AVL.1 na amostra AVL.2, utilizando o modelo Jin, o valor de $f(p)$ é quase $60 \%$ maior do que quando utilizado os valores estimados da própria amostra AVL.2.

A variação no valor da função objetivo foi significativa entre as réplicas em todos os modelos. As amostras AVL.1 e BEN.2 apresentam um menor valor da função objetivo e portanto apresentam um menor desvio dos dados experimentais.

O parâmetro $P_{\max }$ está relacionado à inibição da fermentação pela presença de produto, ou seja, quando o valor de $P$ atinge este limite, a taxa cinética torna-se zero. Analisando-se os resultados deste parâmetro, verifica-se que em alguns casos o mesmo se tornou extremamente elevado. Esta situação anula a inibição por produto no modelo de Ghose. Entretanto, para os modelos de Levenspiel, Lee e Tosetto, o elevado valor de $P_{\max }$ foi acompanhado de um também elevado valor de $\mathrm{n}$ e deste modo a contribuição da inibição por produto ainda existe.

O parâmetro $X_{\max }$, presente no modelo Lee, também foi elevado em todos os casos. Deste modo, não há inibição celular por alta concentração de substrato, já que o expoente $\mathrm{m}$ não foi elevado o suficiente para manter a inibição celular. Este resultado pode ser confirmado ao se comparar os valores praticamente idênticos de $f(\mathbf{p})$ para os modelos de Lee e Levenspiel.

A análise dos resultados, empregando a metodologia proposta, indica que a estimação dos parâmetros foi muito sensível a pequenas alterações nos valores medidos nas variáveis do modelo de fermentação. Estas alterações são causadas pelos próprios erros experimentais, inerentes às medições, ou causadas por fatores não considerados nos modelos cinéticos da literatura.

A Figura 1 apresenta os perfis da concentração de substrato, células e produto das amostras ALV.1 e ALV.2 resultantes do modelo de Jin. 


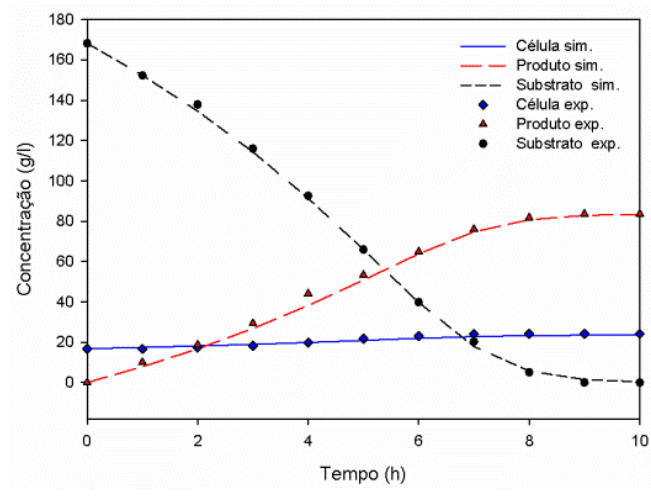

(a)

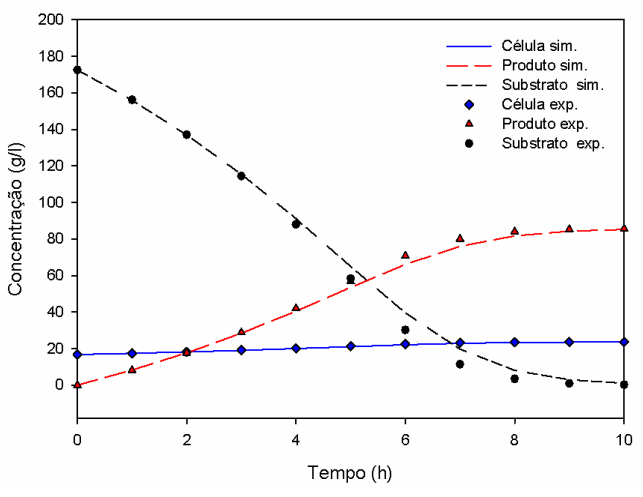

(b)

Figura 1: Simulação do modelo Jin: (a) AVL.1 e (b) AVL.2

Observa-se na Figura 1 que os modelos, para ambas as amostras, apresentar um ajuste satisfatório aos dados experimentais. Outro resultado interessante é que embora os parâmetros Ks e $\mathrm{K}_{1}$ (vide Tabela 2) para - modelo de Jin sejam bastante diferentes, aparentemente o efeito da mudança em um parâmetro compensou a alteração do outro, haja vista que os perfis apresentados nas Figuras 1a e 1b são muito semelhantes.

\section{Conclusão}

A equação cinética que representa o processo fermentativo pode apresentar diversos parâmetros, que podem ser obtidos na literatura ou estimados. Os valores desses parâmetros podem ser influenciados por erros experimentais e também pelas próprias mudanças nas condições operacionais, situação comum nas plantas de fermentação alcoólica.

Nesse trabalho os parâmetros de cinco modelos cinéticos para dois substratos, em duplicata, foram estimados com o objetivo de determinar o efeito dos erros experimentais nos parâmetros cinéticos.

A estimação dos parâmetros foi sensível a pequenas alterações nas variáveis. Portanto desvios nas medidas experimentais ou oscilações no processo podem alterar os valores dos parâmetros obtidos.

\section{Agradecimentos}

Os autores agradecem à CAPES pela bolsa de mestrado e ao CNPq pela bolsa de produtividade DTI.

\section{Referências}

[1] LIMA, U. D. A.; BASSO, L. C.; AMORIM, H. V. D. Produção de etanol. In: AQUARONE, E.; BORZANI, W.; SCHMIDELL, W. (Coord.). Biotecnologia industrial: Processos fermentativos e enzimáticos. São Paulo: Edgar Blucher, v. 3, 2001. p. 1-43.

[2] VASCONCELO, J. N. D. Fermentação Etanólica. In: SANTOS, F.; BORÉM, A.; CALDAS, C. (Coord). Canade-açúcar: Bioenergia, Açúcar e etanol. 2. ed. Viçosa: Folha de Viçosa, 2012. p. 451-487.

[3] BONOMI, A.; SCHMIDELL, W. Modelagem matemática e simulação de processos fermentativos. In: AQUARONE, E.; BORZANI, W.; LIMA, U. A.; SCHMIDELL, W. (Coord.). Biotecnologia Industrial: Engenharia Bioquimica. São Paulo: Edgar Blücher, 2001. v. 2. p. 123-178.

[4] DUTTA, R. Fundamentals of Biochemical Engineering. New Delhi: Ane Books India, 2008.

[5] HISS, H. Cinética de processos fermentativos. In: AQUARONE, E.; BORZANI, W.; LIMA, U. A.; SCHMIDELL, W. (Coord.).. Biotecnologia industrial: Engenharia Bioquimica. São Paulo: Edgar Blücher, 2001. v. 2. p. 93-122.

[6] TOSETTO, G. M. Influência da matéria-prima no comportamento cinético de levedura na produção de Etanol. 95f. Dissertação (Mestrado em Engenharia Química) - Universidade Estadual de Campinas, Campinas, 2002.

[7] Schwaab, M.; Pinto, J. C.. Análise de dados experimentais I: Fundamentos de estatistica e estimação de parâmetros. Rio de Janeiro: e-papers, 2007. v. 1. 